Revista Educación 25(1): 67-79, 2001

\title{
LA ADECUACIÓN CURRICULAR EN MATEMÁTICA
}

Nayibe Tabash Blanco

Resumen: La temática acerca de la adecuación curricular es de gran interés para los profesionales de la Educación. El reto de lograr aprendizajes que atiendan la individualidad en los estudiantes, pone de manifiesto la necesidad de que dichos aprendizajes sean significativos, lo cual implica necesariamente procesos de contextualización y docentes capaces de desarrollar un currículo de manera creativa y asumiendo el rol de investigadores en su accionar cotidiano.

El presente artículo trata de dar a conocer la forma en que la adecuacion curricular puede verse desde una óptica institucional e individual.

\section{Introducción}

El gobierno que inició funciones el 8 de mayo de 1994 presentó como orientación de sus políticas nacionales, el Plan Nacional de Combate a la Pobreza. Este distribuyó sus acciones alrededor de ejes sectoriales, entre estos se encuentra el Sector Educativo, en el cual está inmersa un área prioritaria denominada Proinfancia, la que tiene como finalidad la creación de oportunidades para los niños y jóvenes de hoy en día. Es desde esta perspectiva que surge el Programa para el Mejoramiento de la Calidad y la Educación y Vida en Comunidades Urbano Marginales, llamadas también Comunidades de Atención Prioritaria (PROMECUM).

PROMECUM tuvo como objetivo el "mejoramiento de la calidad de la educación y vida de los niños y niñas, mediante la concentración de diversos sectores y el desarrollo de procesos educativos pertinentes y de calidad, optimizando los métodos más efectivos y los recursos humanos y materiales con que cuenta el Ministerio de Educación Pública y las instituciones del sector educación, que coadyuvan al mejoramiento educativo" (Hernández:1994:7). Además, se promueve el desarrollo integral de los niños en cuatro dimensiones: cognoscitiva (corresponde a la adquisición de conocimientos de calidad), socioafectiva (donde se fortalece la autoestima), psicomotriz y espiritual (involucra los valores propios de nuestra cultura).

Uno de los aspectos que toma en cuenta PROMECUM es un componente 
denominado adecuación curricular, el cual se define "como un mecanismo flexible que permite la modificación, el incremento, el cambio y la disminución temporal de los elementos básicos en el programa de estudio, como los objetivos y los contenidos de aprendizaje y los mecanismos de evaluación, para poder responder a los niños y niñas con necesidades educativas especiales, niños con discapacidades o que hayan avanzado por diferentes niveles sin dominar efectivamente los contenidos de aprendizaje y sin haber logrado los objetivos de niveles anteriores" (Hernández:1996:16).

Este trabajo tiene como objetivo fundamental dar a conocer un modelo de adecuación curricular en el área de la matemática, el cual es el producto final de un trabajo de investigación que se realizó, con el fin de fortalecer la adecuación curricular a nivel institucional, en un centro de Educación Primaria.

El artículo consta de dos apartados. El primer apartado aborda el procedimiento metodológico, algunos resultados que ayudaron a sustentar el modelo y las conclusiones que se obtuvieron.

En el segundo apartado se presenta el modelo de adecuación curricular, el cual se elabora tomando en cuenta los resultados de la investigación.

\section{Procedimiento metodológico, resultados y conclusiones}

La investigación se realizó en una institución educativa de carácter público, ubicada en el sector oeste de San José, Pavas. La institución educativa pertenece al circuito 05 de la Regional de San José.

Se escogió la institución educativa en mención porque reunió las siguientes características: mayor número de docentes y estudiantes, facilidad de acceso a la información y permanencia en el programa desde que éste se inició.

Se recolectó información acerca del conocimiento que tenía el docente, el administrador educativo y el equipo interdisciplinario, con respecto al proceso de adecuación curricular que describe PROMECUM. La población participante estuvo conformado por 62 educadores pertenecientes a una institución de enseñanza primaria del Cantón de San José, distrito de Pavas, circuito 05, Dirección Regional de San José. La información se recolectó utilizando los siguientes instrumentos:

a. Un cuestionario dirigido a todos los docentes de I y II ciclos: Este instrumento permitió la recolección de información acerca del concepto que tiene el educador del proceso de adecuación curricular que contempla PROMECUM, los beneficios que el programa le brinda al docente para realizar la adecuación curricular, las áreas en que se aplicó el proceso, estrategias que utiliza el docente para aplicar la adecuación curricular y por último logros y limitaciones que se obtienen al ejecutar el mismo.

b. Entrevista realizada al administrador educativo y al equipo interdisciplinario (Orientador, Trabajador Social y Psicólogo): En cuanto al administrador educativo la entrevista contempló los siguientes aspectos: características de la población estudiantil, concepto de adecuación curricular que se tiene en la institución educativa. La entrevista que se le hizo al equipo interdisciplinario se centró en lo siguientes aspectos: labor que efectúa el equipo interdisciplinario como apoyo al docente, orientaciones que le brindan al maestro en la programación, ejecución y evaluación del proceso de adecuación curricular.

c. Observaciones realizadas a diferentes docentes que ejecutan la adecuación curricular. Las observaciones realizadas fueron de gran ayuda para visualizar la operacionalización del proceso de adecuación curricular en el aula 
escolar, de ellas se extrajeron elementos importantes tales como: tipo de adecuación curricular que se aplica, ubicación en el aula de los niños que la reciben, métodos y técnicas y recursos utilizados, uso del espacio y del tiempo, así como características del grupo observado.

Los resultados más relevantes obtenidos en la investigación son los siguientes:

Se evidencia que las materias en que se aplicó mayormente la adecuación curricular fueron Matemática con un $51,43 \%$ y Español con un 28,57\%, mientras que Estudios Sociales ocupa un tercer lugar con un 20\%.

- La mayoría de los docentes consideró que al ejecutar el proceso de adecuación curricular en su aula, toman en cuenta aspectos didácticos tales como: uso de fichas, guías de trabajo, material fotocopiado, folletos. Además, para ello se utiliza el trabajo grupal, en parejas, el dirigido por el maestro o con la guía de otro niño y trabajo extraclase. El aspecto socio afectivo en el alumno se estimuló a través del trabajo en grupo y de la incentivación que el docente le daba al estudiante para que este siguiera adelante con el proceso educativo.

- $\quad$ Algunas de las estrategias que utiliza el docente al aplicar adecuación curricular son las siguientes:

* Trabajar con los objetivos y contenidos no alcanzados por el niño.

* Modificar pruebas escritas y orales.

* Trabajar con el niño en forma individual.

* Ubicar al niño en lugares estratégicos: cerca de un compañero más adelantado o cerca del escritorio del maestro.

* Utilizar material didáctico pertinente.

* $\quad$ Brindarle al niño prácticas para que las realice en el hogar.
* Mostrarles afecto y aprecio por el trabajo que realiza.

* Guardar estrecha comunicación entre padre de familia, maestro y alumno.

- De las observaciones que se realizaron y las notas crudas obtenidas se evidencia los siguientes resultados:

* La lección no se inicia con una actividad motivadora, el cierre por su parte es utilizado para la revisión del trabajo que el niño realiza.

* La metodología empleada en el trabajo de aula es tradicional: predomina la utilización de la pizarra, se aplica únicamente la técnica expositiva, inexistencia de material audiovisual, la participación del estudiante es limitada.

* La organización del espacio es tradicional: los niños se encuentran ubicados en hileras.

* Los niños que requieren adecuación curricular están distribuidos en diferentes lugares, en su mayoría se encuentran ubicados cerca del escritorio del docente, esto obedece, según el criterio del maestro, a la pronta atención que le pueda brindar al estudiante.

* Hay un predominio de la evaluación sumativa, dejando de lado la formativa. El área en que se encuentra el mayor número de estudiantes aplazados es Matemática, y se ubica principalmente en el nivel de cuarto grado.

Algunas de las conclusiones obtenidas son las siguientes:

La mayoría de los docentes (88,56\%) consideran que la aplicación del componente de adecuación curricular ayuda a los estudiantes, ya que permite apoyar al niño para que logre obtener los contenidos básicos de acuerdo con el nivel en que se encuentra, además promueve mayores oportunidades al estudiante para superarse. 
- Se evidencia que la adecuación curricular se dirige a ayudar a los estudiantes con necesidades educativas especiales, así como aquellos con algunas discapacidades o que hayan avanzado por diferentes niveles sin dominar los contenidos de aprendizaje y sin haber logrado los objetivos de niveles anteriores.

- $\quad$ El área temática que se debe fortalecer es Matemática y en un nivel de cuarto grado.

- Se debe trabajar con una metodología más activa, con el fin de incentivar y lograr una mayor participación de los estudiantes en el proceso de enseñanza y aprendizaje.

- La adecuación curricular que se realiza es en un nivel de aula y obedece a un tipo de adaptación individualizada.

\section{Modelo de adecuación curricular}

Para el planteamiento del modelo de adecuación curricular se tomaron en cuenta los resultados obtenidos en la investigación, así como una detallada revisión bibliográfica acerca del tema de modelos curriculares. Los hallazgos encontrados que alimentaron la propuesta son los siguientes:

- La materia en que se aplicó mayormente la adecuación curricular fue Matemática.

- La metodología empleada en el trabajo de aula es tradicional.

- Hay un predominio de la evaluación sumativa.

- $\quad$ El área en que se encuentra el mayor número de estudiantes aplazados es Matemática y se ubica principalmente en el nivel de cuarto grado.

El modelo de adecuación curricular propuesto va dirigido a implementarse a nivel institucional debido a que se toma en cuenta el entorno comunal e institucional, las necesidades y expectativas que presentan los estudiantes de los centros educativos. El modelo propuesto presenta rasgos del enfoque socioreconstructivista debido a que predominan los siguientes elementos:

- Se enfatiza el contenido como un elementos esencial que debe incorporar los aportes de la cultura sistematizada y cotidiana.

- La metodología promueve el proceso de socialización: trabajo grupal, análisis de problemas y la investigación, entre otros.

- Se le da importancia al contexto sociocultural ya que valora la cultura cotidiana como elemento fundamental del currículo.

- Se evalúa tanto el producto como el proceso.

- $\quad$ El docente va a ser facilitador en el proceso educativo y el estudiante es un ente activo, creador y crítico de su propia realidad.

Además se presenta algunos rasgos del enfoque psicologista debido a que también se encuentran rasgos tales como:

- Interesan los procesos del estudiante, sus necesidades e intereses.

- $\quad$ Los recursos se utilizan como un medio para estimular el desarrollo de habilidades, destrezas y actitudes en los alumnos.

- $\quad$ Se propician estrategias metodológicas activas. (Díaz:1995).

Por otra parte, el modelo propuesto adopta el concepto de adecuación curricular que Lucarelli define como "una estrategia posible de mejoramiento de la calidad de la educación, que trata de poner en manos del docente, herramientas que le permitan seleccionar creativamente elementos de las distintas fuentes curriculares, organizar y 
utilizar recursos en forma nueva y original, donde el docente pueda acercarse fácilmente a la realidad del alumno y de la comunidad, con el fin de adecuar a ellos el curriculum". (Citado por Hernández: 1995:5).

El modelo de adecuación curricular se sustenta en cuatro grandes interrogantes.

\section{¿Qué hay que enseñar?}

Esta interrogante se desprende de la Política Educativa y las disposiciones que proceden de la Administración Pública, en donde se establecen cuáles son las enseñanzas mínimas y contenidos básicos, así como los componentes curriculares de obligado cumplimiento.

El modelo curricular que se propone se fundamenta en la Política Educativa hacia el Siglo XXI, de donde se desprenden los Programas de Estudio, los que a su vez determinan los objetivos y contenidos de cada área básica del currículo .

Debido a que el área de Matemática presentó el mayor índice de estudiantes aplazados en el nivel de cuarto grado, los objetivos y contenidos se dirigen a fortalecer las destrezas, habilidades y aprendizaje de los alumnos de IV grado.

\section{¿Cuándo hay que enseñar?}

Esta tarea consiste en distribuir y organizar el desarrollo de los objetivos y contenidos a lo largo de ciclos y niveles educativos. Para esto se toman en cuenta la secuenciación y temporalización, las cuales ayudan a organizar y desarrollar los contenidos, de modo que asegure el orden lógico de los aprendizajes.

El criterio de secuenciación se apoya en los siguientes aspectos:

- $\quad$ Adecuación de los contenidos a los conocimientos previos de los educandos.
Es necesario identificar lo que saben los alumnos y encontrar puntos de conexión con los nuevos conocimientos que van a ofrecer.

- Presentación de acuerdo con la lógica de la disciplina seleccionada, en este caso el área de Matemática.

- Elección de un contenido organizador: Se elegirá un contenido organizador con el cual se estructuran los contenidos de apoyo o de soporte.

- Delimitación de los ejes de contenidos: Estos ejes proporcionarán el hilo conductor de la secuenciación, los temas e ideas que se presenten.

- Adopción de una estructura fácil de modificar: Con esto se desea la modificación y secuenciación de los objetivos y contenidos planteados en el modelo.

- Cada uno de los objetivos y contenidos del área de Matemática del nivel de cuarto grado, se priorizaron y secuenciaron mediante la información que aportaron los docentes de cuarto grado del centro educativo donde se realizó la investigación, con el fin de que los maestros sean los que determinen las necesidades inmediatas del programa escolar. También se tomó en cuenta los estándares de matemáticas dados por el Consejo Nacional de Profesores de Matemáticas de los Estados Unidos (National Council of Teachers, 1989).

\section{¿Cómo hay que enseñar?}

Aquí se determina la metodología, las estrategias pedagógicas, los métodos y las actividades de enseñanza y aprendizaje. Para efectos del modelo de adecuación curricular éstas se plantean tomando en cuenta los objetivos y contenidos seleccionados en el área de Matemática, los mismos se fortalecerán mediante estrategias, métodos y actividades que involucren la participación del estudiante y del docente. 
Según Díaz-Barriga Frida (1995:117) "existen diversas formas de organizar y estructurar un curriculum, de acuerdo con el tipo de plan curricular que adopte el diseñador, ya sea lineal, modular o mixto". Para efectos del modelo que se propone se tomará en cuenta el plan modular. El módulo se compone de un conjunto de actividades de capacitación profesional y de una o varias unidades didácticas que proveen al alumno de la información necesaria para desempeñar una o varias funciones profesionales. Además, el plan modular permite combinar diversas estrategias, métodos y actividades que fortalezcan el proceso de enseñanza y aprendizaje en forma creativa y dinámica.

La estrategia metodológica que se utilizó para llevar cabo el plan modular fue la elaboración y aplicación de tres módulos, los cuales contienen temas que presentan un contenido teórico pertinente junto con algunas actividades didácticas.

El primero lleva como título Sistema de Numeración, el segundo Operaciones Fundamentales y el tercero Resolución de Problemas.

\section{¿Qué, cómo, y cuándo evaluar?}

El modelo curricular propuesto evalúa los contenidos y los objetivos seleccionados en el área de Matemática, para ello se utilizarán diversas estrategias y procedimientos de evaluación, considera como parte del proceso de enseñanza y aprendizaje al educando, al educador y al mismo modelo que se plantea. El mismo toma en cuenta la evaluación inicial, la formativa o de proceso y la sumativa o de producto. La evaluación inicial se manifiesta a partir del momento en que se seleccionaron los objetivos y contenidos del área de Matemática. La evaluación formativa está presente cuando se realice cada una de las actividades, esto permite que las estrategias que no han sido eficaces puedan ser modificadas en el proceso de implementación. Por otro lado, la evaluación sumativa se percibirá cuando los estudiantes logren con éxito resolver las actividades propuestas.

Como se menciona anteriormente, la estrategia metodológica que se utiliza para llevar a cabo el plan modular, es la elaboración de tres módulos temáticos en la asignatura de Matemática. Cada módulo se dirige hacia un área específica a saber: Sistema de Numeración, Operaciones Fundamentales y Resolución de problemas.

Cada módulo está estructurado de forma que primero se presente el contenido teórico - para información del docente- y luego las actividades pedagógicas. Además se incorporan algunos ejercicios complementarios que pueden ser de ayuda para fortalecer los contenidos. En algunos casos, los fines didácticos se pueden lograr si las actividades pedagógicas son alcanzadas antes de que el contenido teórico del módulo sea expuesto a los estudiantes.

A continuación se presentan algunas actividades didácticas que forman parte de cada uno de los módulos elaborados.

\section{Módulo No. 1 Sistemas de Numeración}

En este módulo se desarrollan actividades didácticas para desarrollar los siguientes contenidos: decena de millar, valor posicional y algoritmo de la suma.

Actividad No. 1 dígitos".

Participo en el juego "Encuentra los

El objetivo de este juego es el de identificar el valor posicional o relativo de los números. Las cantidades con dígitos ausentes sirven para probar si los niños comprenden las operaciones aritméticas de la suma, la resta, la multiplicación y la división. 
Descripción de juego:

Se deben recortar los números que se encuentran en un tablero (ver figura 1).

Estos se colocan en los espacios en blanco que contienen las operaciones dadas en la ficha adicional (ver figura 2). Cada niño tendrá su tablero y ficha correspondiente.

- El estudiante que logre colocar en el menor tiempo posible, el número correcto en cada una de las casillas de las diversas adiciones, será el ganador. (Kerr:1987:127).

Figura 1

\begin{tabular}{|c|c|c|c|c|}
\hline 0 & 0 & 0 & 0 & 0 \\
\hline 1 & 1 & 1 & 1 & 1 \\
\hline 2 & 2 & 2 & 2 & 2 \\
\hline 3 & 3 & 3 & 3 & 3 \\
\hline 4 & 4 & 4 & 4 & 4 \\
\hline 5 & 5 & 5 & 5 & 5 \\
\hline 6 & 6 & 6 & 6 & 6 \\
\hline 7 & 7 & 7 & 7 & 7 \\
\hline 8 & 8 & 8 & 8 & 8 \\
\hline 9 & 9 & 9 & 9 & 9 \\
\hline
\end{tabular}

Figura 2

\begin{tabular}{|c|c|c|c|c|c|c|c|c|}
\hline \multicolumn{9}{|c|}{ "Encuentra dígitos" } \\
\hline 2 & 5 & 1 & 8 & & 5 & 1 & 73 & \\
\hline+ & 3 & $\square$ & 3 & & + & & $\square 4$ & \\
\hline $\bar{\square}$ & 8 & $\frac{5}{2}$ & $\frac{3}{1}$ & & $\bar{\square}$ & 2 & $5 \square$ & \\
\hline 2 & 1 & $\square$ & 0 & 2 & 7 & 5 & 2 & 8 \\
\hline+ & & 4 & $\square$ & 3 & + & $\square$ & $\square$ & 3 \\
\hline $\bar{\square}$ & 2 & 0 & 2 & 5 & 7 & 9 & 7 & $\square$ \\
\hline
\end{tabular}

\section{Actividad No.2}

Participo en el juego "Operaciones con la tabla del cien".

El objetivo de este juego es reconocer los valores de posición o valores relativos al resolver operaciones de suma y resta en la tabla del cien.

Cada participante debe tener un tablero que contenga los números del 1 al 100 (ver figura 3).

Descripción de juego:

Señale en el tablero cualquier número en la mitad superior de la tabla, ejemplo el número 25. Descanse cualquier objeto sobre tal número. (Ver figura 3).

Con la otro objeto señale el número al que se movería si sumara 3 al número anterior, en el caso del ejemplo sería el número 28. ¿En qué dirección se desplazó.

- $\quad$ Señale ahora el número al que se movería si resta 3 unidades al número anterior, en el caso del ejemplo ya citado el resultado será el número 25 . ¿En qué dirección se desplazó?

Señale el número al cual se movería si sumara 10 al número original (35). Determine el camino más corto.

- ¿Qué ocurre con los números 20 y 30? ¿Qué sucede cuando se suma el número 10 a un número cualquiera? (El resultado se encuentra en la misma columna del número original, en la próxima fila hacia abajo. El camino más corto es directamente hacia abajo).

- Sume ahora un número más complicado al 25, ejemplo el 34.

- $\quad$ Primeramente señale el número 25.

- $\quad$ Luego utilice el dígito de las decenas del 34, es decir el 3, para sumar 3 decenas o un total de 30 unidades al 25. Debe moverse directamente hacia abajo a partir 
del 25. A medida que se va moviendo debe ir diciendo en voz alta la cantidad total sumada: 10, 20, 30". Al cabo de haber completado tres movimientos hacia abajo, se encontrará en el número 55.

- Utilice un dígito de las unidades del 34, es decir 4, para sumar 4 unidades al 55. Debe moverse cuatro encasillados a la derecha del 55. A medida que se va moviendo debe ir diciendo en voz alta la cantidad total sumada: " 1,2 , 3, 4”. Al terminar debe encontrarse en el número 59.

- $\quad$ YYa se ha completado la suma! Note que $25+34=59$.

- $\quad$ Trate de sumar $25+37$ del mismo modo. Notará que en este caso tiene que llegar al final de una fila y tendrá que mover al primer número de la izquierda de la próxima fila. Esto corresponde al proceso de sumar agrupando cantidades.

Por otra parte, la resta resulta mucho más difícil que la suma para la mayoría de las personas. Debe cerciorarse que los niños pueden realizar con facilidad los ejercicios de suma antes de intentar los ejercicios de resta. La resta requiere que se desplacen en las direcciones opuestas a las anteriores, es decir, hacia arriba y hacia la izquierda.

- $\quad$ Reste 13 de 27. Se moverá un encasillado hacia arriba a partir del 27 (terminará en el 17) y luego 3 encasillados a la izquierda (terminará en el 14).

- $\quad$ Trate de restar 25 al número 82. Señale el 82. Muévase hacia arriba: "10, 20”. Luego hacia la izquierda 1, ha llegado al final de la fila.

- Para proseguir muévase a la fila superior en su extremo derecho y continúe moviéndose hacia la izquierda: 2, 3, 4, 5. Ha llegado al número 57.

- Practique utilizando otros números. (Kerr:1987:210).
Figura 3

\begin{tabular}{|l|l|l|l|l|l|l|l|l|l|}
\hline 1 & 2 & 3 & 4 & 5 & 6 & 7 & 8 & 9 & 10 \\
\hline 11 & 12 & 13 & 14 & 15 & 16 & 17 & 18 & 19 & 20 \\
\hline 21 & 22 & 23 & 24 & 25 & 26 & 27 & 28 & 29 & 30 \\
\hline 31 & 32 & 33 & 34 & 35 & 36 & 37 & 38 & 39 & 40 \\
\hline 41 & 42 & 43 & 44 & 45 & 46 & 47 & 48 & 49 & 50 \\
\hline 51 & 52 & 53 & 54 & 55 & 56 & 57 & 58 & 59 & 60 \\
\hline 61 & 62 & 63 & 64 & 65 & 66 & 67 & 68 & 69 & 70 \\
\hline 71 & 72 & 73 & 74 & 75 & 76 & 77 & 78 & 79 & 80 \\
\hline 81 & 82 & 83 & 84 & 85 & 86 & 87 & 88 & 89 & 90 \\
\hline 91 & 92 & 93 & 94 & 95 & 96 & 97 & 98 & 99 & 100 \\
\hline
\end{tabular}

\section{Módulo No. 2 Resolución de Problemas}

Este módulo tiene como objetivo fomentar el interés por la resolución de problemas matemáticos mediante el uso del método heurístico.

En este se presenta la información teórica acerca del Método Heurístico, así como algunas actividades didácticas para la puesta en práctica del mismo.

\section{El Método Heurístico}

El método heurístico aparece en mayor o menor grado en todos los métodos activos. En este método, el alumno debe descubrir el esfuerzo de sus actitudes creadoras. Este método pretende resolver un problema utilizando cuatro etapas:

Entender el problema

En esta parte el maestro formula preguntas, tendientes a lograr que el niño enfoque su atención en la información y en las condiciones dadas en el problema.

Se plantea: ¿Cuáles elementos tiene el problema? y ¿Cómo está organizado cada elemento?

Se desglosa el contenido del texto para saber lo que contiene el problema. A medida que los niños van adquiriendo experiencia en la resolución de problemas, se deben 
ir convirtiendo en los autores de éstos. Esta práctica desarrolla la capacidad para identificar la información importante.

Diseñar un plan para la resolución del problema

El maestro debe dar a conocer al estudiante el mayor número de estrategias de resolución y debe conseguir que el estudiante sea consciente de la estrategia que está a punto de aplicar.

Algunas estrategias heurísticas para resolver problemas matemáticos son las siguientes:

- $\quad$ Estrategia 1: Uso del dibujo, figura o gráfica. El problema se plantea en forma gráfica y la solución queda revelada casi inmediatamente al ser trazadas ciertas líneas auxiliares.

- Estrategia 2: Búsqueda de supuestos ocultos. Los problemas que se prestan a la aplicación de esta estrategia desarrollan la creatividad y la imaginación.

Ejemplo: Colina abajo, en la nieve se ven dos surcos que son las huellas de un par de esquís. Uno de los surcos pasa por un lado del árbol y el otro surco por el otro lado de ese mismo árbol. Encuentre diez explicaciones para este hecho.

- Estrategia 3: Construcción de una tabla. La tabla clasifica y organiza la información de manera que se pueda trabajar más eficiente y rápidamente.

- Estrategia 4: Actuar el problema. Aquí se sugiere dramatizar la situación problemática que se presenta.

Aplicar el plan y resolver el problema escogido.
Repasar la solución y el problema mismo

En esta etapa se realizan dos procesos importantes:

a) Se repasan cuidadosamente los pasos en la resolución del problema. Algunas preguntas que se pueden hacer son las siguientes: ¿Qué hizo para resolver el problema?, ¿le parece a usted que esa respuesta es correcta?, ¿podrá haber otra respuesta correcta además de esa? Las intervenciones tienen la intención de lograr que el estudiante se detenga a examinar en sus propios pensamientos y en la manera en que pensó la resolución del problema.

b) Se crean variaciones del problema que acaba de ser resuelto o crear problemas totalmente nuevos, sugeridos por los estudiantes. Aquí se plantean interrogantes en las cuales se cambien los elementos del problema o se plantea buscando otra solución. (Buján:1995).

\section{Actividad No.1}

- Formar grupos por medio de colores. Se les reparte a cada estudiante un color y luego se agrupan con los estudiantes que tienen el mismo color.

- Cada grupo lee las etapas de resolución de problemas que la maestra le entregó en una hoja poligrafiada.

- La maestra reparte a cada grupo el siguiente problema:

Observe el siguiente cuadro:

\begin{tabular}{|l|c|c|c|}
\hline Carlos &, & I & $* * *$ \\
\hline Ana &, & I I & $* * *$ \\
\hline Juan &, & I I I & $* * * *$ \\
\hline Niños & Paletas & Bastones & Confites \\
& $\$ 15 \mathrm{c} / \mathrm{u}$ & $\mathrm{c} / \mathrm{u}$ & $\$ 5 \mathrm{c} / \mathrm{u}$ \\
\hline
\end{tabular}


Tres niños fueron a la pulpería y compraron diferentes dulces. Carlos llevaba 70 colones, Ana 35 colones y Juan 100 colones.

- $\quad$ En forma oral conteste las siguientes preguntas:

* ¿Cuánto dinero gastó cada niño?

* ¿ ¿Les sobró dinero a cada uno o no les alcanzó?

- $\quad$ En grupo se formulan diferentes preguntas sobre lo que les solicita el problema (primera etapa).

Cada grupo expone las preguntas que formularon.

- Entre todos contestan oralmente las preguntas que se formularon acerca del problema.

Cada grupo de alumnos diseña un plan para solucionar el problema entregado por la maestra (segunda etapa).

- Cada grupo copia en una hoja de papel periódico blanco la solución que encontraron al problema (tercera etapa).

- Cada grupo expone la explicación que hicieron para resolver el problema.

- Los niños contestan las preguntas que la docente les expone:

* Qué estrategias utilizaron para resolver el problema?

* ¿ ¿ Cómo se aseguran que la respuesta es correcta?

* Si Carlos hubiera comprado más paletas, ¿cree usted que le alcanzaría el dinero?

* ¿Qué hubiera comprado Juan con la planta que le sobró?

- Cada grupo comenta acerca de la actividad realizada y le entrega a la maestra la opinión por escrito. (Buján:1987:25-28).

\section{Módulo No. 3. Operaciones Fundamentales}

El objetivo de este módulo es reafirmar el algoritmo de la resta, la multiplicación y la división en cantidades hasta 99999.

Actividad No.1

\section{Participar en el juego "Ordenemos operaciones".}

Procedimiento del juego:

- Todos juntos deben trabajar en la resolución de los ejercicios que se plantean en cada una de las franjas de papel que el maestro previamente ha confeccionado. (ver figura 4).

- $\quad$ El alumno que logre resolver más rápidamente cada una de las franjas será el ganador. (Kerr:1987:50).

Figura 4

\begin{tabular}{|l|l|l|l|l|l|}
\hline 3 & $\mathrm{x}$ & 6 & $:$ & 2 & $=$ \\
\hline
\end{tabular}

\begin{tabular}{|l|l|l|l|l|l|l|l|}
\hline 5 & $\mathrm{x}$ & 7 & - & 3 & + & 4 & $=$ \\
\hline
\end{tabular}

Actividad No. 2

meta".

Participar en el juego "Llegando a la

Procedimiento del juego:

- $\quad$ Formar parejas de estudiantes.

- Se establece el orden de participación de los estudiantes. 
Cada pareja de estudiantes escoge un sobre. Este contiene dos divisiones, las que deben ser resueltas por los grupos de estudiantes. (ver figura 5).

- Cada pregunta que logren acertar tendrá un valor de dos puntos.

- El grupo que obtenga mayor puntaje en un tiempo definido será el ganador.

Figura 5

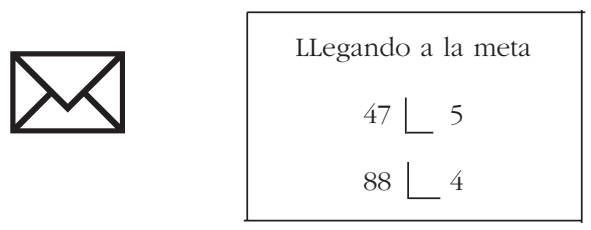

Actividad No. 3

tiplicar".

Participar en el juego "Jugando a mul-

Procedimiento del juego:

- $\quad$ Los estudiantes se turnan para jugar.

- $\quad$ En su turno, cada jugador escoge dos números de la reserva de multiplicandos. (ver figura 6).

- Encontrar el producto de los dos números escogidos.

- Localizar en el tablero el número que está más cercano al producto y colocar una ficha o chapa sobre éste. (ver figura 7).

- Encontrar en el tablero el número que está más cercano al producto.

- $\quad$ El primer jugador en colocar cuatro fichas o chapas en una hilera horizontal, vertical o diagonal es el ganador.

- Los números del tablero están redondeados a la decena más cercana.
Figura 6

Reserva de multiplicaciones

$3-23-31-47-16-18-17-59-13$

Figura 7

\begin{tabular}{|r|r|r|r|r|c|}
\hline 140 & 1460 & 180 & 940 & 360 & 1830 \\
\hline 370 & 12 & 131 & 24 & 510 & 220 \\
\hline 750 & 1321 & 10 & 213 & 90 & 32 \\
\hline 710 & 560 & 340 & 124 & 352 & 70 \\
\hline 290 & 5 & 100 & 23 & 40 & 30 \\
\hline 50 & 9 & 21 & 1000 & 610 & 400 \\
\hline
\end{tabular}

Actividad No.4

saber".

Participar en el juego "Los naipes del

Procedimiento del juego:

- Dos estudiantes se turnan para jugar.

- En su turno, cada jugador escoge un número del tablero. (ver figura 8).

- Luego, elige un naipe, el cual contiene los números del 1 al 9 .

- $\quad$ El número elegido del tablero deberá dividirse entre el número que se encuentra en la carta de naipe escogida.

- $\quad$ El primer jugador que logre resolver cinco divisiones en el menor tiempo posible es el ganador.

Figura 8

\begin{tabular}{|r|r|r|r|r|}
\hline 30 & 100 & 40 & 80 & 20 \\
\hline 80 & 120 & 70 & 600 & 50 \\
\hline 48 & 59 & 64 & 342 & 1245 \\
\hline 3654 & 45 & 679 & 221 & 444 \\
\hline 20 & 90 & 130 & 30 & 10 \\
\hline 555 & 450 & 2357 & 12 & 578 \\
\hline
\end{tabular}




\section{Comentario}

La adecuación o adaptación curricular es un proceso que tiene como finalidad lograr aprendizajes pertinentes, significativos y de calidad, acorde con los requerimientos de las personas y de la realidad de su entorno próximo, en un tiempo y cultura determinados. Por ello, en el contexto del desarrollo del currículo oficial esta condición se trata de garantizar a través de la necesidad que tienen todos los centros de elaborar proyectos educativos, donde se contextualice el currículo oficial en función de las características de cada institución educativa y donde se articule la acción educativa con el equipo docente, así como garantizar la adecuada coherencia de los contenidos educativos a lo largo de la escolaridad, donde se genere un proceso prudente y realista que sirva para detectar y priorizar los principales aspectos que deben cambiarse.

Por lo anterior, es necesario que toda institución de Educación Primaria realice un diagnóstico educativo donde se perciba la realidad comunal, institucional y de aula, con el fin de que estructure un modelo de adecuación curricular que visualice a todos los alumnos en función del progreso que han realizado con respecto a su nivel inicial, con el fin de que este responda a la diversidad y a la individualidad, principalmente en un área de estudio tan problemática como lo ha sido la matemática.

\section{Referencias bibliográficas}

Arias, F. Curriculum y Adecuación Curricular. Ministerio de Educación Pública. Costa Rica. 1993.

Bolster y otros. Matemática, conceptos $y$ práctica. Editorial Scott Foreman and Company. Publicaciones México. 1982.

Buján, V. Número y Forma 4. Farben. Grupo Editorial Norma. San José, Costa Rica.
Buján, V. 1983. Resolución de problemas de matemática en la Educación Primaria. Editorial Alma Mater. San José, Costa Rica.

Carvajal, C. Lineamientos para el diseño curricular. Material mimeografiado. Universidad de Costa Rica. 1997.

Centro Nacional de Recursos para la Educación Especial. Las adaptaciones curriculares y la formación del profesorado. España. 1988.

Díaz-Barriga F. y otros. Metodología del diseño curricular. Editorial Trillas. Capítulo V. 1995.

Espinoza, E. El proceso de elaboración y desarrollo del Programa para el Mejoramiento de la Calidad de la Educación y Vida en Comunidades Urbano Marginales. Ministerio de Educación Pública. San José, Costa Rica. 1995.

Fernández, L. "El perfil de la escuela: su adecuación a condiciones de desigualdad a condiciones de marginalidad". En Revista Latinoamericana de Innovación en Educación. 1991.

Gimeno, J. Comprender y transformar la enseñanza. Editorial Morata. Madrid. Capítulos VIII y IX. 1996.

Hernández, H. Fortalecimiento de los Centros de Recursos para el Aprendizaje de Escuelas Urbano Marginales. Ministerio de Educación Pública. San José, Costa Rica. 1995.

Hernández, H. Orientaciones para la contextualización curricular en las escuelas que niños provenientes de comunidades urbano marginales. Ministerio de Educación Pública. San José, Costa Rica. 1995. 
Hernández, H. La contextualización y la adecuación curricular: Estrategias para alcanzar la pertinencia de la educación en las escuelas urbano marginales. Ministerio de Educación Pública. San José, Costa Rica. 1996.

Hernández, P. Diseñar y enseñar. Teoría y Técnicas de la programación y del proyecto docente. Narcea-ICE, Madrid. 1989.

Jiménez, F. "Adecuación Curricular. Sugerencias para el trabajo en el aula”. Material Mimeografiado. 1993.

Jiménez, M. Adecuaciones Curriculares recomendadas por la Asesoría Nacional de
Problemas de Aprendizaje. Ministerio de Educación Pública. 1996.

Kerr, J y otros. Matemática para la familia. Editorial U.C. Printing Departament. University of California. U:S:A. 1987.

Ministerio de Educación y Ciencia. Los alumnos con necesidades educativas especiales. Secundaria obligatoria. España. 1992.

Pernudt.V. Propuesta: Estilos de aprendizaje en grupos marginales. San José, Costa Rica. 1989.

Retana, C. Análisis del curriculum en una escuela con características de marginidad. San José, Costa Rica. Tesis. 1987. 